\title{
Application of a pressure measuring film for pressure observation in overarm polishing
}

\author{
Michael F. Benisch ${ }^{1, a}$, Oliver Faehnle ${ }^{2}$, and Rolf Rascher ${ }^{1}$ \\ ${ }^{1}$ Institute for Precision Manufacturing and High-Frequency Technology, Deggendorf Institute of Technology DIT, Dieter-Goerlitz- \\ Platz 1, 94244 Deggendorf, Germany \\ ${ }^{2}$ FISBA OPTIK AG, Rorschacherstrasse 268, 9016 St. Gallen, Switzerland
}

\begin{abstract}
In previous publications the inhomogeneous pressure transfer through the polishing tool onto a glass surface could be shown. This experiment shows polishing trials with different polishing materials and the differences in the homogeneity of the pressure transfer through them. Only the properties of the material will be discussed explicitly, the change of the tool constitution through the process will be part of further publications.
\end{abstract}

\section{Introduction}

Previous observations have shown the pressure distribution between the polishing pad and the glass surface. Inhomogeneities in the pressure distribution could also be shown in the plane state. [1]. The pressure during polishing is a relevant process parameter in the Preston equation [2] and directly influences the friction acting in the process [3]. It could also be shown that tilting of the glass surface causes an increase in pressure on the lower side and a decrease in pressure on the upper side. The use of a tool changes the surface of the polishing foil, glass abrasion and cerium oxide are deposited in the pad. For this reason new, non-dressed polishing pads were used and their material properties investigated. The materials used are LP66, LP87 and GR35 (see tab. 1 and fig. 2). A polishing felt was also used for comparison purposes in the case of a flat surface (see fig. 5). It was not used for experiments on the inclined surface.

\section{Setup of the experiments}

In the experiment, the discoloration of a pressure-sensitive foil (4LW, Fujifilm) under the application of force is recorded using a polishing tool. The polishing tool has a diameter of $30 \mathrm{~mm}$ and thus a circular area of about $700 \mathrm{~mm}^{2}$. A polishing pad or felt (see fig. 1, fig. 2 and 4) is part of this tool. The pressure sensitive foil is applied between tool and glass surface. The glass surface is lying straight or is tilted (approximately $30^{\circ}$ ). The force is applied via the lever of an overarm polishing machine, which is additionally loaded with a weight of $1.25 \mathrm{~kg}$, see fig. 1. Pressure measuring films of the type 4LW (Fujifilm) are used. The details of the application of these films and their limitations are described in [1].

\footnotetext{
a e-mail: michael.benisch@th-deg.de
}

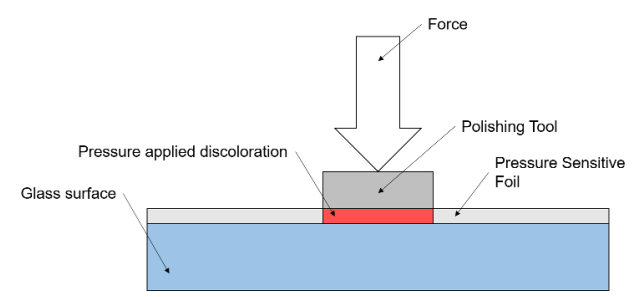

Figure 1. Experimental setup for the case of a plane glass surface: Under the influence of a force, a pressure occurs in the contact area between the polishing pad, pressure measurement film and glass surface. Under this pressure the pressure measuring film changes colour.

\section{Basic Material Properties}

The properties of the polishing foils are shown in table 1 . The films are polyurethane films, which are partly filled with zirconium-oxide (GR35) or cerium-oxide (LP66). The material LP87 has no filler. They are standard material for the production of glasses, crystals and ceramics. The material properties in tab. 1 are manufacturer's (Universalphotonics) data. The approach to determine the modulus of elasticity via tensile tests does not work due to the properties of the polishing pads. Inhomogeneities and porous material areas act as fractures. Therefore the results of tensile tests are poorly comparable.

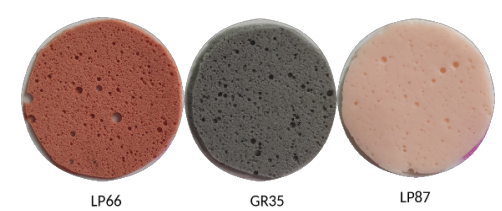

Figure 2. Photography of the polyurethane films used. 
Table 1. Important technical parameters of the polishing pad materials used. The higher the durometer Shore A value, the more resistant the material is to penetration and the harder it is.

\begin{tabular}{|l|c|c|}
\hline Material & Density $\left[\mathrm{g} \mathrm{m}^{-3}\right]$ & Durometer Shore A \\
\hline LP66 & 0.35 & 66 \\
GR35 & 0.59 & 90 \\
LP87 & 0.43 & 96 \\
\hline
\end{tabular}

The results of previous investigations, which were carried out with a comparable polishing tool, clearly show that the pressure distribution on the surface is not homogeneous for plane and tilted surfaces. In particular, if the glass lens and thus the polishing pad is tilted, significant deviations from a homogeneous pressure distribution arise [1]. For the following investigations a new polishing pad was used to see the influence of the material.

\section{Results}

\subsection{Flat Surface}

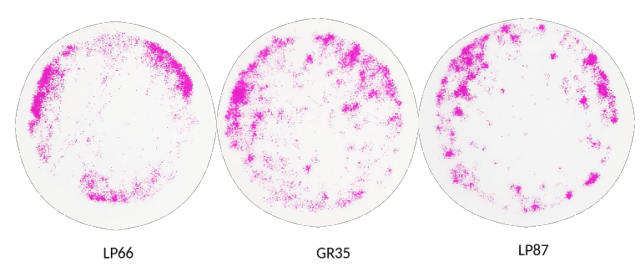

Figure 3. Discoloration of the film after pressure loading on a non-tilted surface.

All three materials show a pressure increase in the edge area. The two harder materials (GR35 and LP87) show additional local pressure increases over the entire contact area between polishing pad and pressure measurement film. The visual impression the pressure distributions is very similar. The somewhat softer LP66 shows a lower tendency to pressure increases (see fig. 3). The cause of these two effects is currently unclear. There may be an influence by the tool shape or the as-new condition of the polishing pad.

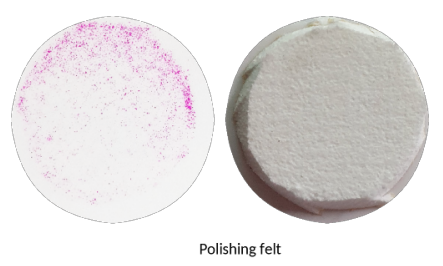

Figure 4. Discoloration of the film after pressure loading on a flat surface with a polishing felt (left) and the polishing felt (right).

If one compares the films with the polishing felt, it is noticeable that the polishing felt has a much more even pressure distribution (see fig. 4). In particular, hardly any pressure increases occur. Pressure marks are present on the entire surface. The discoloration of the pressure measurement film is low (compared to the discoloration caused by the polishing pad). There is a small effect to a one-sided discoloration, which may be caused by the tool shape.

\subsection{Tilted Surface}

Even with a tilted surface, there is a tendency for the two harder materials to have local pressure increases. With the harder materials there is a more extensive pressure distribution. The LP66 material seems to have the tendency for local pressure increases to a lesser extent (see fig. 5), but also shows traces of excessive pressure. All materials show a higher pressure load on the side of the polishing pad which is downwars lying (see fig. 5). The result from [1] is therefore confirmed.

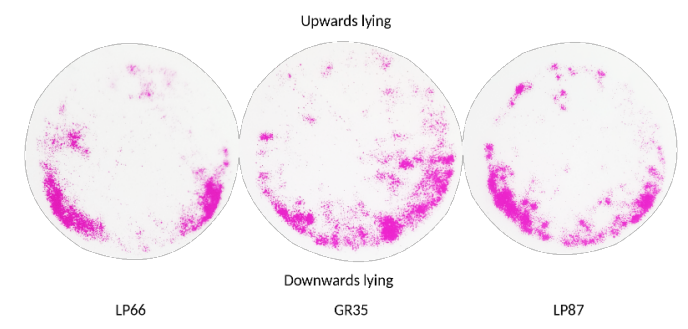

Figure 5. Discoloration of the film after pressure loading on a tilted (approximately $30^{\circ}$ ) surface.

\section{Conclusion}

The present tests show the basic behaviour of different polishing pads under pressure. The already mentioned change of the pressure distribution due to a tilting of the glass lens [1] could be confirmed. For further investigations the influence of the dressing of the tool and its wear condition and their influence on the pressure distribution is of iterest. The effect of a pressure increase at the corners could not be observed with a used tool (compare source). When tilted, the materials all show a tendency to local pressure increases. Furthermore, there is a need for a way to monitor the pressure during a process at different points of the tool, especially for the case of a tilted surface to polish (sperhical lens).

\section{References}

[1] Benisch, M. F.; Faehnle, O. Rascher R.; Bogner, W.; Force and Pressure Analysis during Overarm Polishing POM 2020, (2020)

[2] Preston F. W.; The theory and design of plate glass polishing machines Journal of the society of glass, 214-256 (1926)

[3] Belkhir N.; Bouzid D.; Herold V.; Determination of the Friction Coefficient During Glass Polishing Tribology Letters, 55-61 (2009) 